\title{
Commentary
}

\section{Adolescent Cannabinoid Use and Cognition; Unexpected Results from a Rat Model of Cannabinoid Self-Administration}

\author{
Barry Setlow*,1,2,3,4 and Jennifer L Bizon ${ }^{1,2,4}$ \\ 'Department of Psychiatry; University of Florida College of Medicine, Gainesville, FL, USA; ${ }^{2}$ Department of Neuroscience; University of Florida \\ College of Medicine, Gainesville, FL, USA; ${ }^{3}$ Department of Psychology; University of Florida, Gainesville, FL, USA; ${ }^{4}$ Center for Addiction Research and \\ Education, University of Florida, Gainesville, FL, USA
}

Neuropsychopharmacology (2017) 42, 983-984; doi:I0.1038/npp.2016.212; published online 12 October 2016

Cannabis is the most widely used illicit substance in the United States and worldwide, and its use is expected to increase with recent and pending changes in its legal and medical status. Cannabis use during adolescence is of particular concern because of its association with enduring cognitive impairments and overall worse life outcomes. Whether these deleterious outcomes are a direct result of cannabis use, however, remains controversial (Curran et al, 2016). Indeed, many of the genetic and environmental factors that increase the likelihood of drug experimentation (eg, impulsivity, low socioeconomic status, and chronic stress) also predispose individuals to adverse outcomes, even in the absence of drug use. Animal studies to date, however, also show that cannabinoid administration during adolescence can induce cognitive deficits that persist into adulthood. While directly addressing causality, these prior animal studies also have limitations, as they have employed passive drug administration, which fails to model the volitional nature of human drug use. This is an important distinction as passive versus active drug administration can result in unique neural and behavioral consequences. Selfadministration models (in which a response is required in order to receive a drug) better mimic human drug use, but cannabinoid self-administration can be challenging to establish. In this issue of NPP, Kirschmann et al. report the first animal model of adolescent cannabinoid selfadministration. Rats in this study were trained to intravenously self-administer the synthetic CB1 receptor agonist WIN55,212-2 (WIN) in daily sessions over several weeks during mid- to-late adolescence. The rats acquired robust WIN self-administration at levels comparable to those shown previously in adult rats (Fattore et al, 2007).

A primary goal of these studies was to evaluate the effects of adolescent cannabinoid self-administration on adult

\footnotetext{
*Correspondence: Dr B Setlow, Department of Psychiatry, University of Florida College of Medicine, P.O. Box 100256, Gainesville, FL 326I00256, USA, Tel: +352 294 5203, Fax: 352392 9887,

E-mail: setlow@ufl.edu

Received 28 August 2016; revised 2 September 2016; accepted 7 September 2016; accepted article preview 22 September 2016
}

cognitive outcomes. Rats that self-administered WIN during adolescence, together with control rats that had comparable experience self-administering a food reward, were assessed under drug-free conditions on several tests of prefrontal cortex (PFC)-dependent mnemonic function (delayed-response working memory and spatial/object recognition memory tasks). Somewhat surprisingly, cannabinoid selfadministration had no adverse effects on performance across a range of adult time points, and working memory performance was actually enhanced. These findings stand in sharp contrast to the cognitively impairing effects of passive adolescent cannabinoid exposure reported previously, and suggest that the route of administration has a critical role in behavioral consequences of cannabinoids. It is also important to acknowledge, however, that the cumulative doses of self-administered WIN were markedly lower than doses used in prior passive administration studies. Indeed, Kirschmann et al. replicated a previous demonstration of impaired spatial and object recognition memory following passive administration of higher WIN doses (Abush and Akirav, 2012). It is possible that these and similar impairments are due to the increased anxiety reported to follow passive, high dose cannabinoid administration. It remains to be determined whether passive administration of doses more akin to those achieved through self-administration produces such deleterious behavioral effects.

The most surprising result from Kirschmann et al. was the enhanced working memory following adolescent WIN selfadministration. This enhancement was evident both 20 and 60 days following drug cessation, and was accompanied by biochemical evidence for an enduring shift in the normal balance of excitatory and inhibitory signaling in PFC. Prefrontal cortical excitatory and inhibitory circuit and signaling dynamics are essential for working memory, and GABAergic signaling dysfunction in particular is increasingly recognized as a contributor to working memory impairments across a range of conditions (McQuail et al, 2015). In the current study, rats that self-administered WIN showed increased adult PFC expression of the R2 subunit of the $\mathrm{GABA}_{\mathrm{B}}$ receptor $\left(\mathrm{GABA}_{\mathrm{B}} \mathrm{R} 2\right)$, and of the primary 
neuronal transporter for GABA, GAT-1. This GAT-1 upregulation, and consequent increased efficiency in GABA clearance, might be expected to facilitate the sustained pyramidal neuronal activity required for information maintenance, thereby contributing to enhanced working memory performance. The fact that $\mathrm{GABA}_{\mathrm{B}} \mathrm{Rs}$ are localized to both pre- and post-synaptic sites makes it difficult to speculate regarding the specific synaptic consequences of the increased $\mathrm{GABA}_{\mathrm{B}} \mathrm{R} 2$ expression. Nevertheless, this finding certainly suggests that there is increased surface expression of $\mathrm{GABA}_{\mathrm{B}}$ Rs associated with adolescent cannabis use. Despite remaining questions regarding specific mechanisms, the fact that such robust alterations in GABAergic signaling protein expression were present well after cessation of drug use highlights a critical need to better elucidate how cannabis use in adolescents alters PFC GABAergic signaling parameters, and to further define the degree to which such effects endure across the lifetime. Single-cell analyses of PFC circuit dynamics, together with the biochemical and behavioral approaches employed by Kirschmann et al., could help clarify the cellular mechanisms whereby cannabis impacts GABAergic signaling and determine the circumstances under which cannabinoids can benefit (or harm) cognition.

Despite the absence of cognitive impairments resulting from WIN self-administration, the take-home message should not be that adolescent cannabis use is harmless. In addition to GABAergic signaling alterations, WIN selfadministration also produced robust cue-induced reinstatement following extinction, which increased in magnitude following extended abstinence (ie, incubation of craving (Pickens et al, 2011)). These latter data highlight the potential of cannabinoid cues to promote continued use throughout the lifespan. Indeed, ongoing consumption may be a key variable in the long-term cognitive consequences of cannabis use (Curran et al, 2016).

The results of Kirschmann et al. represent an important step in modeling adolescent cannabis use and its long-term consequences, but as with any such first step, many questions remain unanswered. For example, it remains to be seen how adolescent WIN self-administration influences affective behaviors (eg, depression- and anxiety-like measures) that are altered by passive administration. In addition, future studies that build on Kirschmann et al. to incorporate a range of drug doses, both sexes, and additional rat strains, will help shape the conclusions regarding the long-term consequences of cannabis use in adolescence. These latter two variables in particular may be relevant for future research in this area, as females may be more sensitive than males to cannabis and cannabinoid administration (eg, (Fattore et al, 2007; Wiers et al, 2016)) and at least one report suggests that adult rats of the strain employed by Kirschmann et al. (Sprague-Dawley) self-administer WIN less readily than other strains (Fattore et al, 2007). These issues notwithstanding, the current results provide a critical advance toward a preclinical research agenda on adolescent cannabis use.

\section{FUNDING AND DISCLOSURE}

The authors declare no conflicts of interest.

\section{REFERENCES}

Abush H, Akirav I (2012). Short- and long-term cognitive effects of chronic cannabinoids administration in late-adolescence rats. PLoS One 7: e31731.

Curran HV, Freeman TP, Mokrysz C, Lewis DA, Morgan CJ, Parsons LH (2016). Keep off the grass? cannabis, cognition and addiction. Nat Rev Neurosci 17: 293-306.

Fattore L, Spano MS, Altea S, Angius F, Fadda P, Fratta W (2007). Cannabinoid self-administration in rats: sex differences and the influence of ovarian function. Br J Pharmacol 152: 795-804.

McQuail JA, Frazier CJ, Bizon JL (2015). Molecular aspects of age-related cognitive decline: the role of GABA signaling. Trends Mol Med 21: 450-460.

Pickens CL, Airavaara M, Theberge F, Fanous S, Hope BT, Shaham Y (2011). Neurobiology of the incubation of drug craving. Trends Neurosci 34: 411-420.

Wiers CE, Shokri-Kojori E, Wong CT, Abi-Dargham A, Demiral Ş, Tomasi D et al (2016). Cannabis abusers show hypofrontality and blunted brain responses to a stimulant challenge in females but not in males. Neuropsychopharmacology 41: 2596-2605. 